\title{
Scholtz, Hanno: Mediensoziologie. Eine systematische Einführung.
}

\author{
Wiesbaden: Springer VS 2020. 330 Seiten. Preis: $€ 19,99$ (e-book)
}

\author{
Helmut Scherer
}

Angenommen: 3. Juni 2021 / Online publiziert: 30. Juni 2021

(C) Der/die Autor(en) 2021

Dieses Buch ist eine Einführung, die sich an Bachelor-Studierende richtet. Aus diesem Grund erscheint es sinnvoll, das Buch aus der Perspektive des Hochschullehrers zu bewerten, der sich fragt, ob er das Werk seinen Studierenden der Kommunikationswissenschaft empfehlen sollte. Die Beschäftigung mit den Perspektiven der Nachbardisziplinen ist gute Tradition in der Kommunikationswissenschaft, die sich ja vielfach als Integrationsdisziplin versteht. Leider geht dabei mitunter der eigenständige Charakter des Faches verloren. Grundsätzlich bleibt es aber zu begrüßen, wenn man sich selbstbewusst, aber auch selbstkritisch mit den relevanten Forschungsarbeiten aus den Nachbardisziplinen auseinandersetzt. Normalerweise bewerten wir in unserer Forschungs- und Lehrtätigkeit Arbeiten nicht auf Basis der Disziplin, aus der sie stammen, sondern auf Basis ihrer Relevanz und Qualität. Wenn Scholtz typisch ist für die Mediensoziologie, dann sieht man dies dort offensichtlich ganz anders. Beiträge aus anderen Disziplinen fasst man dort scheinbar nur mit spitzen Fingern an. Und genau darin liegt das Kernproblem des Buches, Scheuklappen sind selten hilfreich, aber in diesem Fall hoch wirksam.

Ganz offensichtlich bemüht sich der Autor in erheblichem Maße, den Anforderungen an ein Lehrbuch gerecht zu werden. Das Werk wird mit einer Einführung eingeleitet, bei der Scholtz die wichtigsten Begriffe „Soziologie“ und „Medien“ definiert, wobei er sich bei der Soziologie nicht so recht entscheiden mag, seine Position dennoch hinreichend deutlich wird. Medien sind nach seiner Definition „Produzenten abgeleiteter Wirklichkeiten“ (S. 9). Die weiteren zumeist knappen 13 Kapitel folgen dann einem einheitlichen Schema. Sie beginnen mit einem Überblick zu den Inhalten, sie enthalten viele kleine Beispiele und etwas ausführlichere

Prof. Dr. Helmut Scherer $(\bowtie)$

Institut für Journalistik und Kommunikationsforschung, Hochschule für Musik, Theater und Medien Hannover, Expo Plaza 12, 30539 Hannover, Deutschland

E-Mail: helmut.scherer@ijk.hmtm-hannover.de 
Fallbeispiele. Abgeschlossen werden die Kapitel jeweils mit einer Zusammenfassung und Kontrollfragen. Danach wird die zentrale Literatur zum Kapitel aufgeführt und zusätzlich werden Beispiele für mediensoziologische Studien und Lehrbücher gegeben. Außerdem bemüht sich der Autor um ein hohes Maß an Verständlichkeit.

Das didaktische Bemühen um Nachvollziehbarkeit muss man loben, aber unglücklicherweise führt es nicht immer zum Erfolg. So ringt der Autor offensichtlich um eine klare und nachvollziehbare sprachliche Darstellung. Manches Mal wird es aber schlicht unverständlich, manches Mal werden Dinge eher schlecht erklärt, man verliert den Kontext aus den Augen oder die Darstellung ist zu weitschweifig. Manchmal mäandert der Text relativ unfokussiert vor sich hin. Da beginnt man an irgendeinem theoretischen Konzept zu lesen, kommt dann zu einigen Fallbeispielen und beschäftigt sich dann mit etwas, von dem man nicht weiß, warum das in diesem Zusammenhang bedeutsam sein sollte, und der Autor sagt einem auch nicht, warum dies dort relevant ist. So steht im Kapitel über das Internet relativ unvermittelt ein Teilkapitel zum Begriff der Anonymität. Natürlich ist unmittelbar einsichtig, dass Anonymität mit der Internet-Kommunikation etwas zu tun hat, aber eine Verbindung zu den vorher dargestellten theoretischen Positionen und Ansätzen unterbleibt nahezu vollständig. Und es gibt auch Kapitel, die wirklich gar nicht gelungen sind wie Teil-Kapitel 10.4, in dem es um den statistischen Nachweis von Zusammenhängen geht. Ansonsten ist die Darstellung zu Methoden aber durchaus in Ordnung. In Bezug auf den didaktischen Anspruch des Buches fällt die Bilanz also durchwachsen aus, wobei letztlich dennoch die positiven Aspekte überwiegen.

Inhaltlich sieht dies aber anders aus. Auch hier gibt es Stärken und Schwächen. Vieles ist sehr hilfreich und wird vom Autor auch kenntnisreich und profiliert entfaltet. Die Stärke des Bandes zeigt sich, wenn medienbezogene Problemstellungen sehr gut mit dem Rückgriff auf soziologische Theorien und Erklärungsmuster aufgearbeitet werden. Hier wird soziologische Denkweise auch für Bachelor-Studierende aus anderen Fächern nachvollziehbar. Als sehr basale Einführung in die Soziologie funktioniert das Buch also recht gut. Die Medien bleiben aber in der Darstellung allzu häufig auf der Strecke und die Forschungsarbeit der Kommunikationswissenschaft zu den entsprechenden Fragestellungen wird weitgehend ignoriert.

Die Fallbeispiele sind in dem vorliegenden Band ein zentrales didaktisches Mittel, sie sind aber auch der Bereich, in dem Scholtz häufig scheitert. Viele Beispiele haben gar keinen Medienbezug. Dies gilt auch dann, wenn dieser ohne Probleme herstellbar wäre, etwa beim Fallbeispiel zur Lebensstilforschung, wenn Scholtz den Opernbesuch wählt, statt naheliegenderweise die Mediennutzung. Manches Mal erscheint der Medienbezug auch sehr aufgesetzt, wenn es etwa um den FramingBegriff geht und dabei auf den Weinstein-Skandal eingegangen wird. Vollkommen daneben ist Fallbeispiel 26. Wussten Sie schon, dass der Tod von Michael Jackson 2009 eine Revolution im Iran verhindert hat? Nein; ich auch nicht. Scholtz stellt aber ernsthaft die These auf, der Tod von Jackson habe die Aufmerksamkeit der Weltöffentlichkeit von den Unruhen im Iran abgelenkt und die Proteste seien in Folge abgeflaut.

Möglicherweise resultiert der mangelnde Einbezug von Medienthemen bei den Fallbeispielen aus der grundsätzlichen Ignoranz des Autors gegenüber der kommunikationswissenschaftlichen Forschungsliteratur. Dabei wäre es doch so naheliegend. 
Noelle-Neumanns Schweigespirale könnte als gutes Beispiel für das Modell der soziologischen Erklärung gelten. Soziologische Grundbegriffe wie Rolle, System oder Feld könnten am Beispiel der Journalismusforschung gut verdeutlicht werden. Es ist schon fast als Kunststück zu begreifen, dass jemand über die Motivation zur Mediennutzung schreibt und dabei die Uses-and-Gratifications-Forschung vollkommen ausblendet. Auch der Umgang mit dem Begriff Öffentlichkeit ist bedauerlich reduziert und simplifiziert.

Scholtz Medienbegriff führt fast notwendigerweise zu zwei zentralen kommunikationswissenschaftlichen Fragestellungen. Wenn Medien „Produzenten abgeleiteter Wirklichkeiten“ sind, dann wird es hochrelevant, was die Regeln und Bedingungen dieser Ableitung sind und welche Konsequenzen diese haben. Demnach müssten die Ansätze zur Nachrichtenauswahl von besonderer Bedeutung sein - also Gatekeeperund Nachrichtenwerttheorien - ebenso wie die Forschung zur Realitätsvermittlung durch Medien, also etwa die Kultivationstheorie. Eine kurze Abhandlung zur Nachrichtenselektion gibt es immerhin, sie ist aber eher rudimentär. Der Begriff Kultivation taucht aber gar nicht auf und der Name George Gerbner wird nie genannt. Das ist eigentlich inakzeptabel. Man mag nun gegen diese Kritik einwenden, dass es sich hier ja um eine Einführung in die Mediensoziologie handle und nicht um eine Einführung in eine soziologisch fundierte Kommunikationswissenschaft. Aber wie sinnvoll ist eine fachliche Abgrenzung, die zur Ausgrenzung ganzer relevanter Forschungsbereiche führt? Die Antwort ist einfach: überhaupt nicht. Will man ein taugliches Lehrbuch zur Mediensoziologie verfassen, dann hilft es nichts, relativ weitschweifig in die Geschichte soziologischer Grundbegriffe zurückzugehen, aber die dafür relevanten Ansätze, Theorien und Ergebnisse der Kommunikationswissenschaft einfach auszublenden.

Würde ich das Buch also meinen Studierenden empfehlen? Ich denke, eher nicht, zumindest nicht generell. Vielleicht kann man einzelne Studierende, die an bestimmten soziologischen oder medien-soziologischen Fragestellungen interessiert sind, darauf hinweisen. Für die Mehrzahl der Studierenden wird das Buch nicht hilfreich sein.

Funding Open Access funding enabled and organized by Projekt DEAL.

Open Access Dieser Artikel wird unter der Creative Commons Namensnennung 4.0 International Lizenz veröffentlicht, welche die Nutzung, Vervielfältigung, Bearbeitung, Verbreitung und Wiedergabe in jeglichem Medium und Format erlaubt, sofern Sie den/die ursprünglichen Autor(en) und die Quelle ordnungsgemäß nennen, einen Link zur Creative Commons Lizenz beifügen und angeben, ob Änderungen vorgenommen wurden.

Die in diesem Artikel enthaltenen Bilder und sonstiges Drittmaterial unterliegen ebenfalls der genannten Creative Commons Lizenz, sofern sich aus der Abbildungslegende nichts anderes ergibt. Sofern das betreffende Material nicht unter der genannten Creative Commons Lizenz steht und die betreffende Handlung nicht nach gesetzlichen Vorschriften erlaubt ist, ist für die oben aufgeführten Weiterverwendungen des Materials die Einwilligung des jeweiligen Rechteinhabers einzuholen.

Weitere Details zur Lizenz entnehmen Sie bitte der Lizenzinformation auf http://creativecommons.org/ licenses/by/4.0/deed.de.

Prof. Dr. Helmut Scherer ist Professor für Kommunikations- und Medienwissenschaft an der Hochschule für Musik, Theater und Medien Hannover. 